\title{
A method for bearing fault diagnosis of mine hoist using convolutional attention autoencoder
}

Jie Zhang

Anhui University of Science and Technology https://orcid.org/0000-0002-6055-545X

Ke Yang ( $\nabla$ yksp2003@163.com )

Anhui University of Science and Technology

Yuanyuan Jiang

Anhui University of Science and Technology

Ling Xia

Anhui University of Science and Technology

\section{Research}

Keywords: Hoisting bearing, Fault diagnosis, Robustness, Attention mechanism, Autoencoder

Posted Date: July 9th, 2021

DOI: https://doi.org/10.21203/rs.3.rs-678821/v1

License: @ (i) This work is licensed under a Creative Commons Attribution 4.0 International License.

Read Full License 


\title{
A method for bearing fault diagnosis of mine hoist using convolutional attention autoencoder
}

\author{
Jie Zhang ${ }^{1,2,3} \cdot$ Ke Yang ${ }^{1,2,3} \cdot$ Yuanyuan Jiang $^{4} \cdot$ Ling Xia $^{4}$
}

Received: date / Accepted: date

\begin{abstract}
In view of the complex environment and frequent faults in the actual operation of mine hoist, a fault diagnosis method based on Convolution Attention Autoencoder (CAAE) is proposed through theoretical analysis and experimental verification to improve the diagnostic stability of mine hoist under strong noise. First, a CAAE is constructed, which uses a combination of a convolutional neural network ( $\mathrm{CNN})$ and a channel attention module (CAM) to compress and encode the input signal, and then the input signal is reconstructed by a decoder to train the CAAE to extract the original signal fault features. Then, a fault diagnosis classifier is constructed to classify different fault patterns. Finally, experimental validation is performed with the Case Western Reserve University bearing dataset. The results show that the method has a strong feature extraction capability and a high classification accuracy for bearing failure modes compared with existing methods. And the experiments on the application effect of the proposed method in noisy environment are conducted
\end{abstract}

$\bowtie$ Ke Yang yksp2003@163.com

1 State Key Laboratory of Mining Response and Disaster Prevention and Control in Deep Coal Mines, Anhui University of Science and Technology, Huainan 232001, China

2 National \& Local Joint Engineering Research Center of precision coal mining, Anhui University of Science and Technology, Anhui, Huainan, 232001, China

3 Key Laboratory of Mining Coal Safety and Efficiently Constructed by Anhui Province and Ministry of Education, Anhui University of Science and Technology, Huainan 232001, China

4 College of Electrical and Information Engineering, Anhui University of Science and Technology, Huainan 232001, China to verify that the method is highly effective and challenging.

Keywords Hoisting bearing · Fault diagnosis · Robustness · Attention mechanism · Autoencoder

\section{Introduction}

Mine hoist is a crucial equipment connecting ground and underground, and its stable operation is directly related to the safe and efficient production of coal mines (He et al. 2016)(Kou et al. 2020). As a statistical fact, $70 \%$ of the failures of rotating machinery are caused by vibration (Jiang et al. 2013), 30\% of which are caused by bearings ( $\mathrm{Gu}$ et al. 2020). However, the characteristics of vibration signals, such as nonlinearity and nonsmoothness, bring great challenges to signal fault diagnosis. Therefore, it is important to investigate mining hoist bearing fault diagnosis methods for the special working environment of mines.

Since conventional machine learning methods are mostly shallow networks, it is difficult to handle signals with strong noise interference, which limits the ability to extract high-dimensional features (Qi et al. 2017). In recent years, fault diagnosis methods based on deep learning have received wide attention, and the core of deep learning is feature learning, which aims to learn fault features from the input signal by highlevel abstract modeling of the signal through deep networks. To effectively improve the signal-to-noise ratio (SNR) of vibration signals (Hamadache et al. 2017) and extract signal fault features, therefore, CNN (Eren et al. 2019) (Wang et al. 2019) and Autoencoder (AE) (Chen et al. 2017) (Ma et al. 2018) have been widely used for bearing fault diagnosis. Wu et al. proposed 
a fault diagnosis method based on convolutional neural network to solve the extraction of nonlinear signal features by using the powerful feature extraction ability of the one-dimensional convolutional neural network (1D-CNN). Liu et al introduced a multi-task 1D convolutional neural network model to improve the accuracy of the model by using different branches of different tasks to process the fault feature information at individual locations, and the performance of the model was verified by comparing with other networks(Liu et al. 2020). Wen et al. studied a fault diagnosis method for automatic extraction of fault features, which converts $1 \mathrm{D}$ signals into two-dimensional images and uses twodimensional convolutional neural networks (2D-CNN) for feature extraction, eliminating the effects of incomplete manual feature extraction (Wen et al. 2016). Liu et al proposed a CAE method for bearing fault diagnosis using CNN to enhance the feature extraction capability of denoising autoencoders and the effectiveness of the method was verified with bearing and gearbox datasets(Liu et al. 2019). Che et al. extracted the input signal fault features using a denoising autoencoder and input the features into a convolutional neural network model for fault mode diagnosis, which improved the diagnostic performance of the model in a noisy environment(Che et al. 2020). However, the above fault diagnosis method, which cannot effectively extract the key features of the noisy input signal, limits the application of the approach to equipment fault diagnosis in noisy environments.

With the above analysis, the paper has presented a fault diagnosis method using a combination of $1 \mathrm{D}$ convolutional autoencoder (CAE) and channel attention mechanism. First, the attention mechanism is introduced in the CAE network, and the key features of the signal are extracted by the established attention module, and the residual learning is introduced to retain the fault information of the input signal; then, a deep learning classifier model is used to build a deep learning classifier model to classify the extracted fault features to obtain the diagnostic results of the fault patterns. The results of the experimental comparison and analysis verify the good diagnostic performance and stability of the proposed method. The contribution of the innovation of the paper is summarized as follows.

(1) A channel attention module based on attention mechanism and residual theory is designed in this paper for enhancing the overall model for feature extraction of key signals of the input signal.

(2) For the first time, a novel feature extraction method is built by combining the channel attention module with a convolutional autoencoder, which can extract the key fault features in the bearing vibration signal.

(3) The performance of the model in bearing fault diagnosis is further revealed by comparing with four methods in a noisy environment.

The remainder of the paper is broadly structured as follows. In Section II, the design of each module of the convolutional attention autoencoder network and the framework and process of the bearing fault diagnosis method are described in detail. A bearing fault diagnosis the framework was established. Section III presents the data set processing and model evaluation criteria for bearing fault diagnosis experiments. Section IV verifies the performance of the proposed method in the paper through experiments, and analysis and discussion are conducted. Finally, the work of this paper is summarized in Section V.

\section{CAAE model and fault diagnosis process}

\subsection{CAE Module}

An autoencoder is an artificial neural network that learns an efficient representation of the input data by compressing the input into a kind of hidden space for representation and then reconstructing the input data using a decoder (Shao et al. 2017), and the basic structure of is demonstrated in Fig. 1.

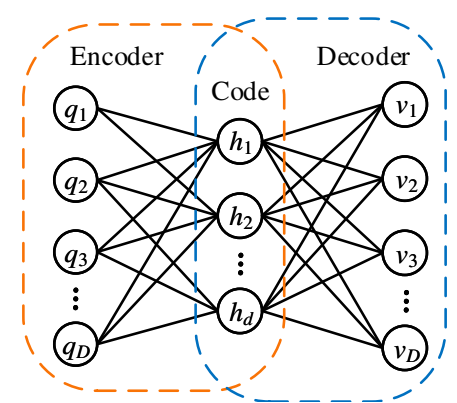

Fig. 1 The structure of an autoencoder

The training sample $\mathbf{Q}=\left[\mathbf{q}_{1}, \mathbf{q}_{2}, \cdots, \mathbf{q}_{m}\right]$ is given, and for each $\mathbf{q}_{n}=\left[q_{1}, q_{2}, \cdots, q_{D}\right]^{T}(n \epsilon[1,2, \ldots, D])$. The input $\mathbf{Q}$ is transformed into the hidden space $\mathbf{H}=$ $\left[\mathbf{h}_{1}, \mathbf{h}_{2}, \cdots, \mathbf{h}_{m}\right]$ by the encoder, where $\mathbf{h}_{n}=\left[h_{1}, h_{2}, \cdots\right.$ ,$\left.h_{D}\right]^{T}$. By the following function transformation

$\mathbf{H}=f\left(\mathbf{W}^{(1)} \mathbf{Q}+\mathbf{b}^{(1)}\right)$

where $f(\cdot)$ is the sigmoid activation function; $\mathbf{W}^{(1)}$ and $\mathbf{b}^{(1)}$ are the weight matrix and bias, respectively.

By using the decoder to reconstruct $\mathbf{H}$ into $\mathbf{V}$, the reconstructed output component $\mathbf{V}=\left[\mathbf{v}_{1}, \mathbf{v}_{2}, \cdots, \mathbf{v}_{m}\right]$, 


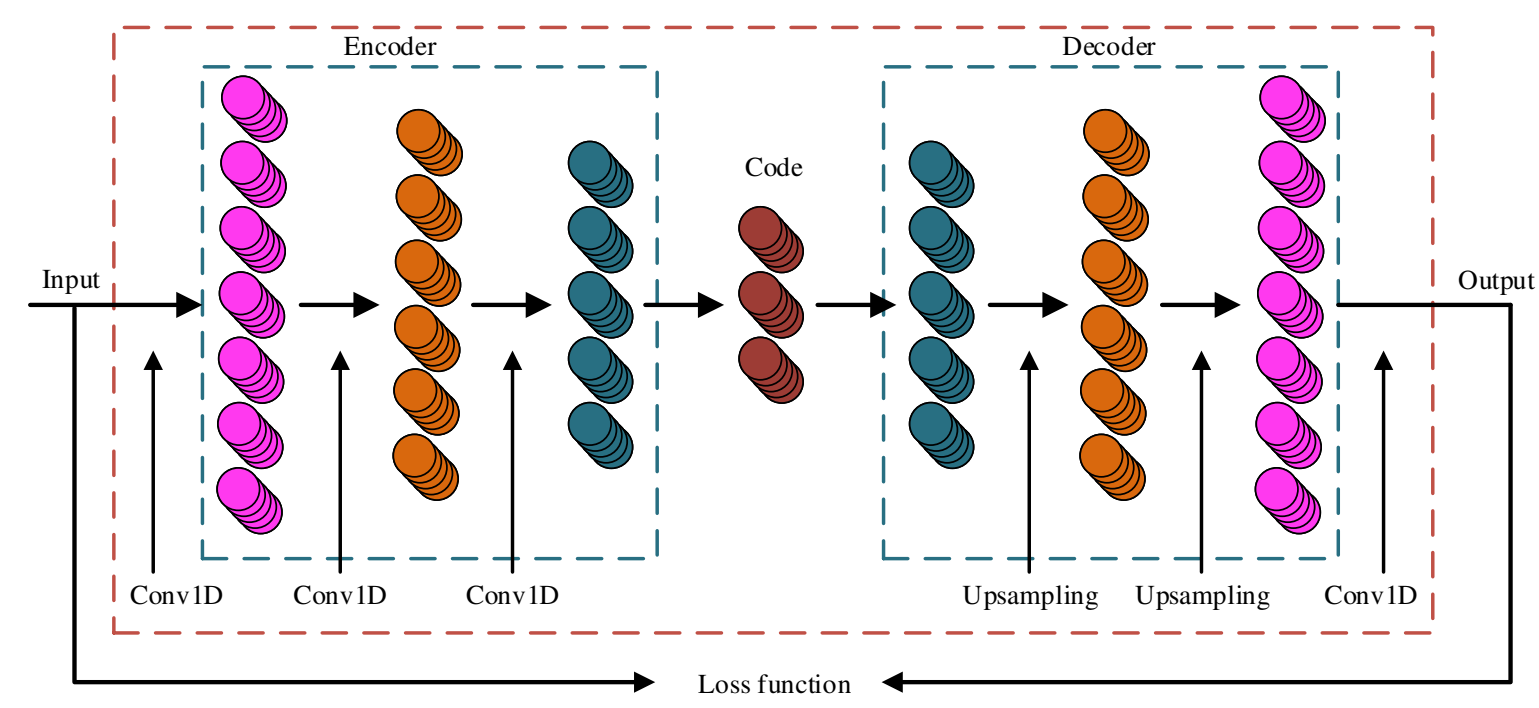

Fig. 2 Convolutional autoencoder basic framework

for each $\mathbf{v}_{n}=\left[v_{1}, v_{2}, \cdots, v_{D}\right]^{T}, \mathbf{V}$ is denoted as

$\mathbf{V}=f\left(\mathbf{W}^{(2)} \mathbf{H}+\mathbf{b}^{(2)}\right)$

where $\mathbf{W}^{(2)}$ and $\mathbf{b}^{(2)}$ are the weight matrix and bias, respectively.

The autoencoder is designed to obtain the optimized encoder weights and bias parameters by training. The mean squared deviation is usually used as the loss function of the autoencoder.

$L_{M S E}=\frac{1}{m} \sum_{i=1}^{m}\left(\frac{1}{2}\left\|\mathbf{v}_{n}-\mathbf{q}_{n}\right\|\right)$

A CAE is composed of a combination of a $1 \mathrm{D}-\mathrm{CNN}$ and an AE, where both the encoder and decoder are built from convolutional layers. The basic framework of the convolutional autoencoder is shown in the Fig. 2. The encoder can use convolution operations to compress the input signal into a specific spatial representation, which is then reconstructed into the input signal using the upsampling operation in the encoder.

\subsection{Channel Attention Module}

By mining the interdependencies among the channel mappings, the limited attention is focused on the focused information, thus saving resources and obtaining effective feature information (Fu et al. 2019).

Throughout the fault feature extraction process of vibration signals, some features may be irrelevant to the fault information and may even obtain a wrong diagnosis. For this reason, this paper introduces channel attention module (CAM) to selectively extract useful channel information and suppress useless channel information by mining the interdependencies between channel mappings, and adaptively learn fault-related features (Xu et al. 2021).

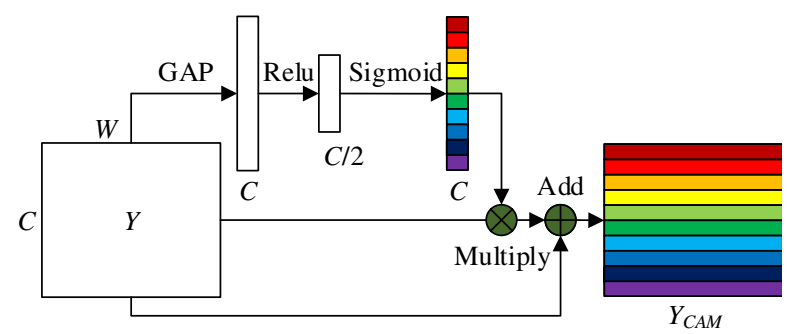

Fig. 3 Channel attention module structure

The detailed idea of the CAM is given in the Fig. 3 . $\mathbf{Y}$ is the feature map of the $1 \mathrm{D}$ convolutional output, and the input $\mathbf{Y}=\left[\mathbf{y}_{1}, \mathbf{y}_{2}, \cdots, \mathbf{y}_{C}\right], \mathbf{y}_{i} \in R^{W \times 1}$, where $C$ is the number of channels, $i \epsilon[1,2, \cdots, C]$, and $W$ is the feature width. $\mathbf{Y}$ is compressed by Global Average Pooling (GAP) to obtain the channel attention component $\mathbf{U}=\left[u_{1}, u_{2}, \cdots, u_{C}\right]$ defined as

$u_{i}=\frac{1}{W} \sum_{j=1}^{W} y_{i, j}$

The $1 \mathrm{D}$ excitation weights $\mathbf{Y}\left(\mathbf{Y}=\left[z_{1}, z_{2}, \cdots, z_{C}\right]\right)$ are learned by training with one-dimensional convolution and fully connected layers to activate each layer of channels, and the formula is

$\mathbf{Z}=\sigma(G(\delta(F(\mathbf{U}))))$

where $F(\cdot)$ and $G(\cdot)$ are the one-dimensional convolution with channel number 1 and the fully connected 
operation with node number $C$, respectively; $\sigma$ and $\delta$ are the Relu and Sigmoid functions, respectively.

The attention to the critical channel domain is enhanced by multiplying the input $\mathbf{Y}$ with the excitation weight $Z$. The Equation is as follows

$\mathbf{M}=\mathbf{M} \times \mathbf{Z}=\left[\mathbf{y}_{1} z_{1}, \mathbf{y}_{2} z_{2}, \cdots, \mathbf{y}_{C} z_{C}\right]$

The idea of residuals is introduced to improve the discriminability of the features while preserving the original information, and the final channel features are obtained as

$\mathbf{Y}_{C A M}=\mathbf{M}+\mathbf{Y}$

\subsection{Convolutional Attention Autoencoder Module}

In this paper, based on the feature extraction capability of 1D-CNN on temporal signals (Wang et al. 2019) and the learning capability of the channel attention mechanism on key features, the one-dimensional CAE is combined with the channel attention module to build a CAAE module to extract the fault characteristics of the input signal.

The structure of the CAAE module is shown in Fig. 4. The encoder part consists of three one-dimensional convolutional layers and three attention modules, and the input signal is compressively encoded by the threelayer convolutional attention module to obtain the fault feature representation of the input signal. The decoder consists of three upsampling layers and three 1D convolutional layers to reconstruct the encoded input signal and gradually recover the feature dimensionality of the input signal. The loss function is used to minimize the error between the reconstructed signal and the reconstructed signal to achieve a functional mapping as a function of the difference between the two.

The parameters of each layer of the CAAE network are configured as shown in Table 1. The input signal sample length is 2048, and the dimension is compressed to 256 by the encoder, and the number of channels is 64 in the hidden space. The decoder is then used to decode and reconstruct it into the input signal itself, thus achieving feature extraction of the input signal.

\subsection{Mine hoist fault diagnosis process}

To achieve mine hoist fault diagnosis, a method based on convolutional attention autoencoder fault diagnosis is proposed, and the overall fault diagnosis method flow chart is shown in the Fig. 5, and the specific diagnosis steps are as follows.

Step1: Obtain the original vibration signal.

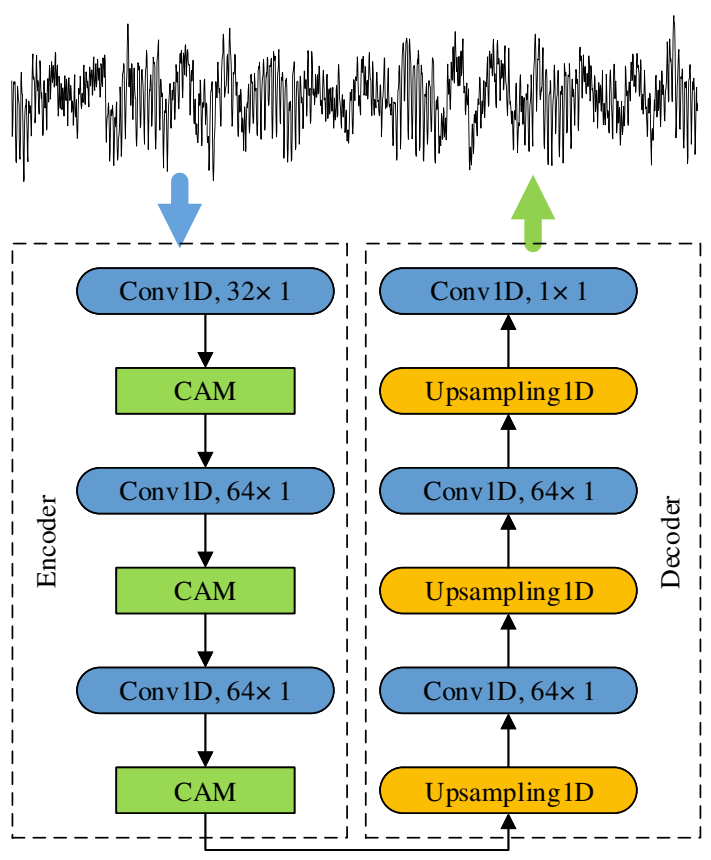

Fig. 4 CAAE module structure

Table 1 Network configuration of CAAE module

\begin{tabular}{ccc}
\hline Network layer & Parameters (filters, kernel size) & Output \\
\hline Input & - & $2048 \times 1$ \\
\hline Conv1D & 32,28 & $1024 \times 32$ \\
\hline CAM & - & $1024 \times 32$ \\
\hline Conv1D & 64,14 & $512 \times 64$ \\
\hline CAM & - & $512 \times 64$ \\
\hline Conv1D & 64,10 & $256 \times 64$ \\
\hline CAM & - & $256 \times 64$ \\
\hline Upsampling1D & - & $512 \times 64$ \\
\hline Conv1D & 64,7 & $512 \times 64$ \\
\hline Upsampling1D & - & $1024 \times 64$ \\
\hline Conv1D & 64,5 & $1024 \times 64$ \\
\hline Upsampling1D & - & $2048 \times 64$ \\
\hline Conv1D & 1,3 & $2048 \times 1$ \\
\hline & &
\end{tabular}

Step2: The original signal is window sliding according to the window length of 2048 and step size of 300 to obtain the data set, and divided into training set and test set, and the sample data are processed by random sorting.

Step3: The training and test sets are normalized by the formula

$x^{\prime}=\frac{x-\mu}{\nu}$

where $x$ is the input data; $\mu$ is the mean of the input data; and $\nu$ is the variance of the input data.

Step4: The training set is fed into a CAAE module to train the autoencoder module, where the autoencoder is trained using an SGD optimizer with a loss function of binary_crossentropy, a batch training volume of 64 , and a total number of iterations of 200 . 


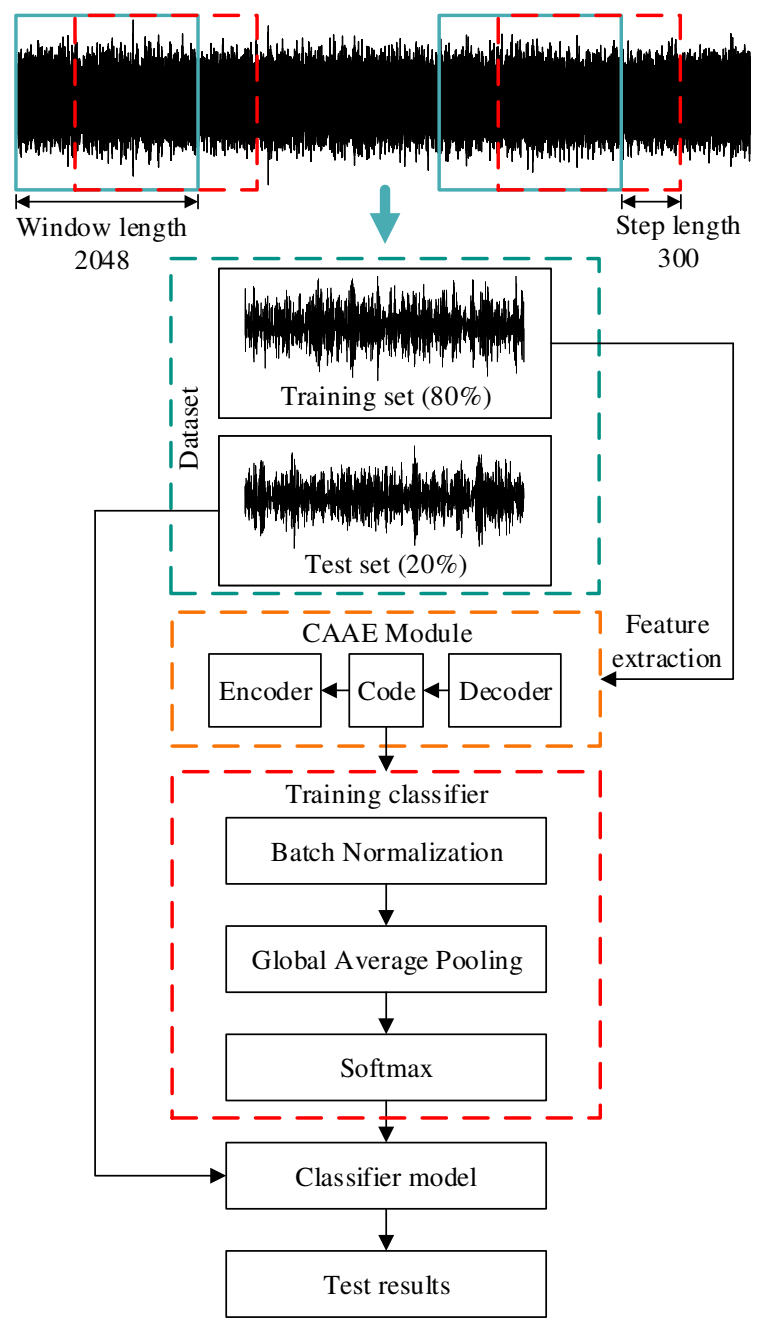

Fig. 5 Overall framework of fault diagnosis

Step 5: The decoder part of the CAAE is removed, and the fault features obtained by the encoder module are input into the batch normalization layer for batch normalization operation.

Step6: The batch normalized features are fed into the GAP layer for global average pooling to reduce the number of training parameters and avoid overfitting.

Step 7: The final classification is performed by softmax classifier.

\section{Experimental Analysis}

In the paper, the proposed CAAE mine hoist bearing fault diagnosis method is implemented in the tensorflow framework of python3. In the training fault diagnosis model, Adam optimizer is used with a learning rate of 0.001 and a batch training volume of 64 .

The model performance was validated using publicly available data on rolling bearings from Case West-
Table 2 Network configuration of CAAE module

\begin{tabular}{cccc}
\hline $\begin{array}{c}\text { Fault } \\
\text { Location }\end{array}$ & $\begin{array}{c}\text { Fault } \\
\text { diameter } / \mathrm{mm}\end{array}$ & $\begin{array}{c}\text { Training set } \\
/ \text { Test set }\end{array}$ & Label \\
\hline Ball & 0.1778 & $320 / 80$ & $\mathrm{~F} 0$ \\
Ball & 0.3556 & $320 / 82$ & $\mathrm{~F} 1$ \\
Ball & 0.5334 & $320 / 81$ & $\mathrm{~F} 2$ \\
Inner race & 0.1778 & $320 / 80$ & $\mathrm{~F} 3$ \\
Inner race & 0.3556 & $320 / 81$ & $\mathrm{~F} 4$ \\
Inner race & 0.5334 & $320 / 74$ & $\mathrm{~F} 5$ \\
Outer race & 0.1778 & $320 / 79$ & $\mathrm{~F} 6$ \\
Outer race & 0.3556 & $320 / 80$ & $\mathrm{~F} 7$ \\
Outer race & 0.5334 & $320 / 82$ & $\mathrm{~F} 8$ \\
Normal & 0 & $320 / 78$ & $\mathrm{~F} 9$ \\
\hline
\end{tabular}

ern Reserve University (LOPARO et al.). The experimental setup is shown in Fig. 6 and consists of a motor, torque sensor, power test meter, and electronic controller involving three types of failures: inner race, outer race, and ball failures. The drive end bearing model SKF6205-RS JEM SKF was used to machine faults in the inner race, outer race and ball of the bearing using electric spark with fault sizes of $0.1778 \mathrm{~mm}, 0.3556 \mathrm{~mm}$ and $0.5334 \mathrm{~mm}$.

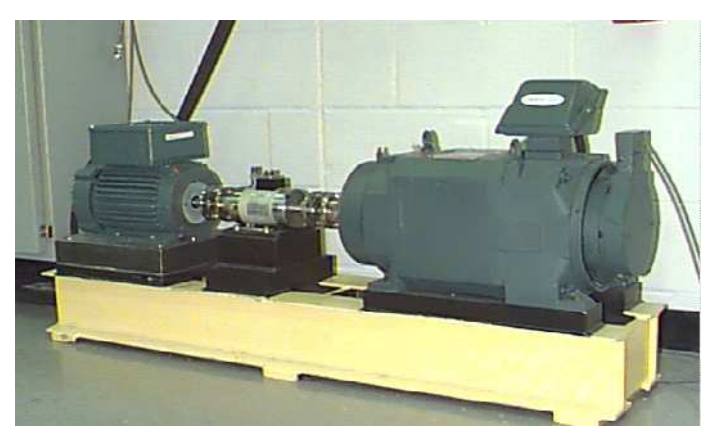

Fig. 6 Bearing test platform

The data used for the experiments were selected from 9 fault modes and normal modes at a motor load of $1 \mathrm{HP}$, a sampling frequency of $12 \mathrm{kHz}$, and an approximate motor speed of $1772 \mathrm{r} / \mathrm{min}$, for a total of 10 fault diagnosis modes, and the time-domain waveforms of the 10 modes are shown in Fig. 7. The used training set and test set experimental data are sampled by sliding window, where the sampling window length is 2048 sample points and the step length is 300 sample points. The data sets were collected for the 10 types of vibration data separately, and 320 training samples were selected for each type, containing a total of 3200 samples, and the test set contained 802 samples, as indicated in Table 2.

The diagnostic performance of the CAAE network is measured by three performance metrics: accuracy Acc, precision Pre and recall $R$ (Zhang et al. 2020), which 

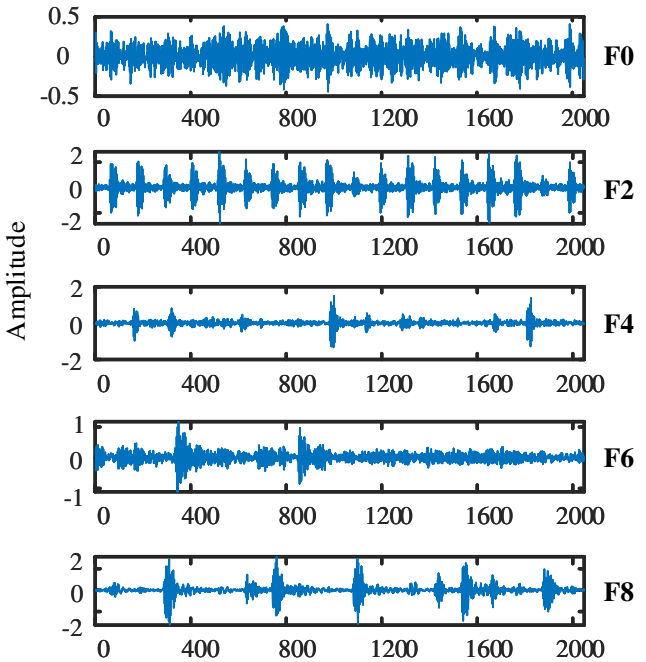

Fig. 710 kinds of vibration signals of bearing failure

are defined as

$A c c=\frac{T P+T N}{T P+F N+F P+T N} * 100 \%$

$P=\frac{T P}{T P+F P} * 100 \%$

$R=\frac{T P}{T P+F N} * 100 \%$

where $T P, F P, T N$ and $F N$ are the number of true positive, false positive, true negative and false negative samples, respectively, and the accuracy, precision and recall are in the scale from 0 to 1 .

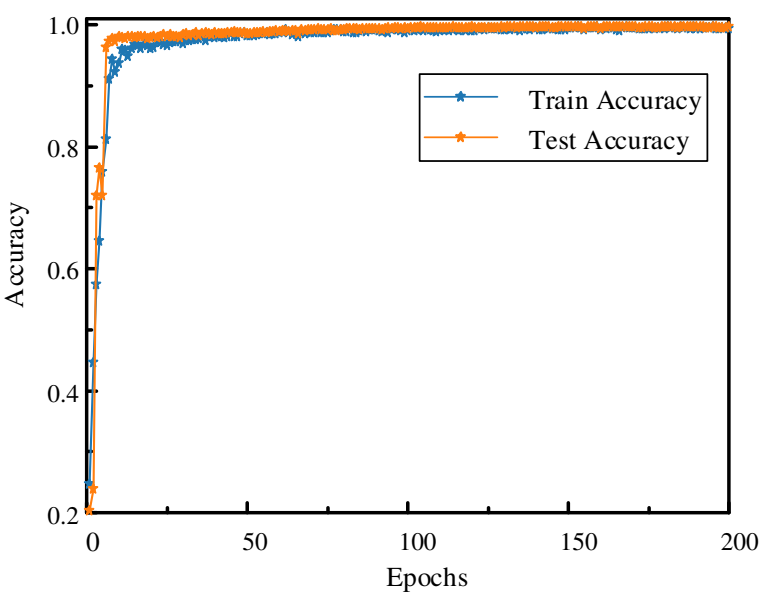

Fig. 8 Accuracy curve of CAAE model

To a more comprehensive evaluation of the model performance, we also conducted stability experiments of
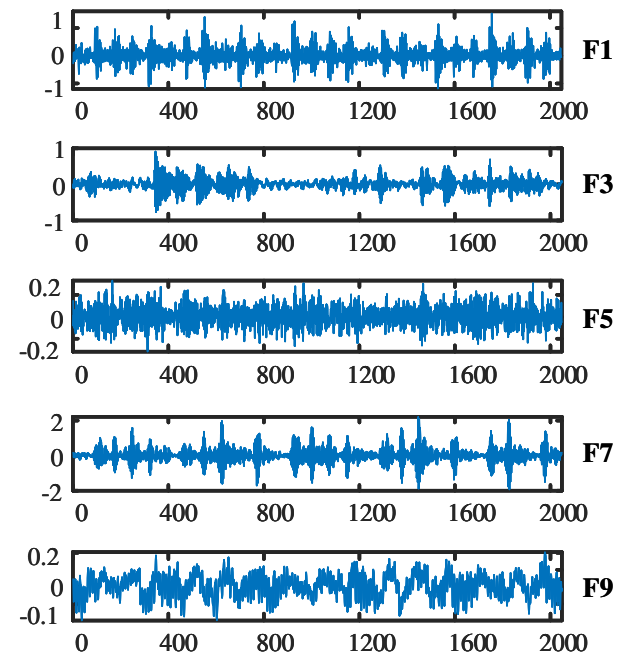

the CAAE model. Gaussian white noise is added to the original signal, and the noise immunity of the CAAE model is verified by comparison experiments. Signal-tonoise ratio is an important index for evaluating signal noise, defined as

$S N R_{d B}=10 \log _{10}\left(\frac{P_{\text {signal }}}{P_{\text {noise }}}\right)$

where, $P_{\text {signal }}$ and $P_{\text {noise }}$ are the power of signal and noise, respectively.

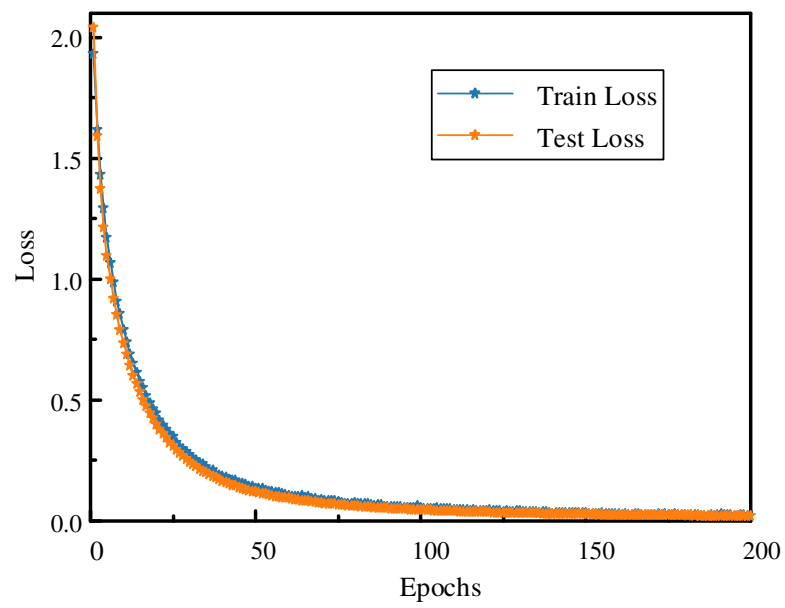

Fig. 9 Loss curve of CAAE model

\section{Experimental results and comparison}

\subsection{Experimental results}

As shown in Fig. 8, the accuracy curves of the CAAE model, the final training accuracy and testing accuracy are $99.34 \%$ and $99.63 \%$, respectively. The training 


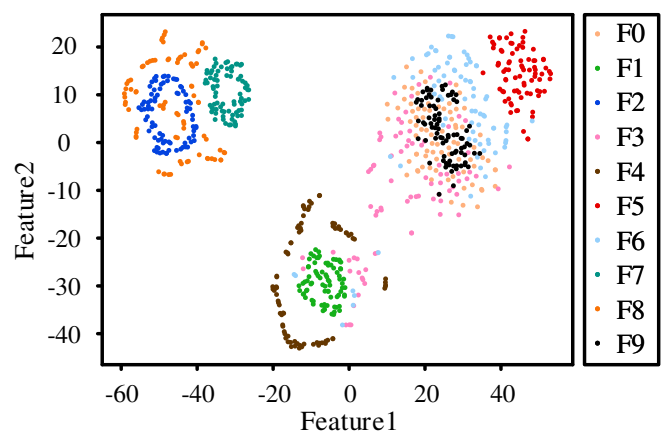

Fig. 10 Visualization of classifier CAAE output features

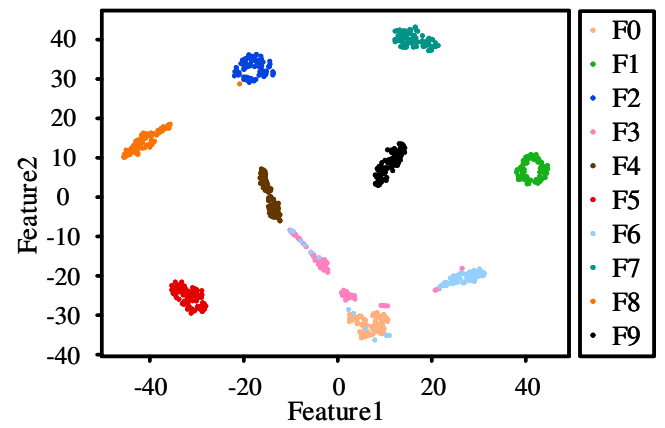

Fig. 11 Visualization of classifier GAP output features

and testing accuracies steadily increased with the number of training iterations during 200 iterations, and the training and testing accuracies converged at a shorter number of iterations, indicating the effectiveness of the proposed method in suppressing the model degradation caused by model overfitting.

From Fig. 9, it is observed that the error curve is gradually decreasing as the iterations increase, and convergence is reached at a shorter number of iterations, indicating that the proposed method converges quickly and steadily.

The output features of the key layers in the CAAE fault diagnosis network are visualized by the t-SNE technique (Zheng et al. 2018), which displays the output features of the convolutional attention autoencoder module, the GAP layer and the classifier in 2D space. The distribution of the fault features in the $2 \mathrm{D}$ space can be clearly seen as shown in Figs. 10. The Fig. 10 shows that there are different degrees of overlap in the $2 \mathrm{D}$ visualization of the output features of the convolutional attention autoencoder module. In Fig. 11, the output features are better classified after the GAP layer. In contrast, the output feature visualization results in a Fig. 12 show that the fault types are better separated. As the results indicate, the method proposed in this paper can effectively diagnose bearing failures.

To better understand the diagnostic accuracy of each failure mode, the confusion matrix was drawn. As shown in the Fig. 13, where F1, F2, F3, F4, F5, F7, F8 and

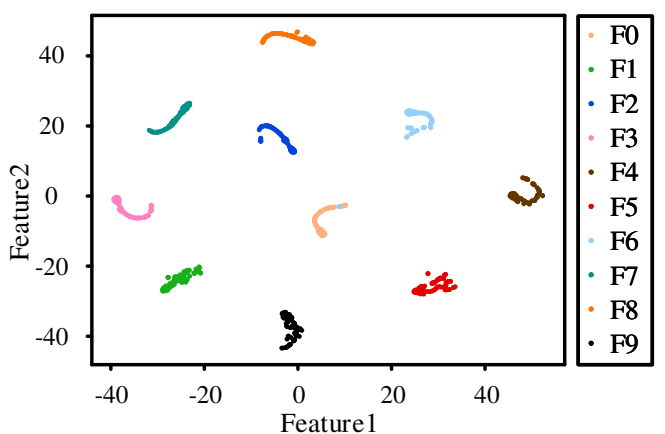

Fig. 12 Visualization of classifier output features

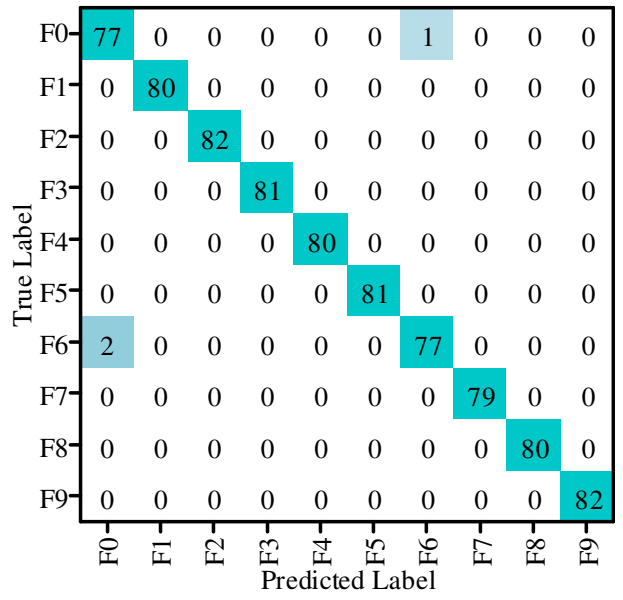

Fig. 13 Confusion matrix of diagnostic results

Table 3 Effect of Convolutional Channel Attention Module on Model Performance

\begin{tabular}{ccccc}
\hline Module Number & Acc (\%) & Pre (\%) & R (\%) & Time (s) \\
\hline CCAM-1 & 88.03 & 83.28 & 88.03 & 0.052 \\
CCAM-2 & 98.50 & 98.62 & 98.50 & 0.075 \\
CCAM-3 & 99.63 & 99.63 & 99.63 & 0.088 \\
CCAM-4 & 99.25 & 99.25 & 99.25 & 0.101 \\
\hline
\end{tabular}

Table 4 Performance comparison of fault diagnosis methods

\begin{tabular}{ccccc}
\hline Method & Acc (\%) & Pre (\%) & R (\%) & Time (s) \\
\hline CAAE & 99.63 & 99.63 & 99.63 & 0.088 \\
CAE & 88.15 & 88.26 & 88.15 & 0.055 \\
1D-CNN & 98.25 & 98.25 & 98.26 & 0.074 \\
CA & 96.13 & 96.20 & 96.13 & 0.101 \\
2D-CNN & 97.01 & 97.09 & 97.01 & 0.120 \\
\hline
\end{tabular}

F9 fault categories can be accurately identified. Overall, the diagnostic accuracy of most of the fault modes in the model network is very close to $100 \%$, indicating that the convolutional attention autoencoder model has good potential for practical applications. 
Table 5 Stability comparison of fault diagnosis methods

\begin{tabular}{|c|c|c|c|c|c|c|}
\hline SNR & Indicators & CAAE & CAE & 1D-CNN & $\mathrm{CA}$ & 2D-CNNN \\
\hline \multirow{4}{*}{$-6 \mathrm{~dB}$} & $\operatorname{Acc}(\%)$ & 90.52 & 72.69 & 74.19 & 85.66 & 67.08 \\
\hline & Pre $(\%)$ & 90.55 & 71.11 & 73.53 & 85.60 & 67.98 \\
\hline & $\mathrm{R}(\%)$ & 90.52 & 72.69 & 74.19 & 85.66 & 67.08 \\
\hline & Time (s) & 0.080 & 0.053 & 0.064 & 0.087 & 0.121 \\
\hline \multirow{4}{*}{$0 \mathrm{~dB}$} & $\operatorname{Acc}(\%)$ & 97.76 & 80.30 & 93.39 & 93.52 & 89.15 \\
\hline & Pre (\%) & 97.79 & 79.62 & 93.45 & 93.53 & 88.66 \\
\hline & $\mathrm{R}(\%)$ & 97.76 & 97.76 & 93.39 & 93.52 & 89.15 \\
\hline & Time (s) & 0.081 & 0.051 & 0.066 & 0.087 & 0.428 \\
\hline \multirow{4}{*}{$6 \mathrm{~dB}$} & $\operatorname{Acc}(\%)$ & 99.50 & 83.41 & 97.26 & 97.26 & 96.51 \\
\hline & Pre $(\%)$ & 99.51 & 83.33 & 97.28 & 97.63 & 96.50 \\
\hline & $\mathrm{R}(\%)$ & 99.50 & 83.42 & 97.26 & 97.63 & 96.51 \\
\hline & Time (s) & 0.082 & 0.052 & 0.065 & 0.094 & 0.120 \\
\hline
\end{tabular}

\subsection{Experimental comparison}

The encoder part of the CAAE network designed in this paper is implemented using a convolutional channel attention module (CCAM) composed of stacked convolutional layers and CAM. To verify the effect of the number of CCAM on the performance of the model, four network structures are built: CCAM-1, CCAM2, CCAM-3 and CCAM-4, respectively, followed by a number representing the number of CCAM.

Table 3 shows that the accuracy of the model increases with the increase in the number of CAAM. It shows that stacking the number of modules can be achieved to improve the performance of the fault diagnosis model. When the number of modules is increased to four, the fault diagnosis rate of the model remains basically unchanged. However, this increases the testing time of the model. Therefore, in this paper, the encoder part of the autoencoder is built using three CAAM module.

To verify the performance of the proposed method in this paper, four metrics of accuracy, precision, recall and time of CAE, 1D-CNN, convolutional attention mechanism $(\mathrm{CA})$ and $2 \mathrm{D}$ convolutional neural network (2D-CNN).

As shown in Table 4, we can see that the accuracy, precision and recall of the proposed method in this paper are $99.63 \%$, which is higher than the other four networks. Comparison with the CAE method shows that adding the channel attention mechanism can effectively extract the fault features of the input signal, and comparison with the CA method shows that the feature extraction method through the autoencoder can more comprehensively extract the features of the bearing fault signal. And compared with CNN model, which shows that simple convolutional neural network can obtain higher accuracy, but not higher accuracy. And the accuracy of $2 \mathrm{D}-\mathrm{CNN}$ is $1.24 \%$ lower than that of $1 \mathrm{D}$ $\mathrm{CNN}$, indicating that the process of converting $1 \mathrm{D}$ sig- nals to $2 \mathrm{D}$ signals through $2 \mathrm{D}-\mathrm{CNN}$ loses some useful information related to faults.

To verify the noise immunity of the CAAE, it was tested against CAE, 1D-CNN, CA and 2D-CNN at signalto-noise ratios of $-6 \mathrm{~dB}, 0 \mathrm{~dB}$ and $6 \mathrm{~dB}$. The experimental results are shown in Table 5. The accuracy, precision and recall of the proposed method in this paper are better than the other methods under three signal-tonoise ratios. Among them, the accuracy of $90.52 \%$ can still be obtained when the signal-to-noise ratio is $-6 \mathrm{~dB}$, which indicates that the proposed method has stronger noise immunity than the other four methods. When the signal-to-noise ratio is $0 \mathrm{~dB}$ and $6 \mathrm{~dB}$, the accuracy only decreases by $7.24 \%$, which is the smallest decrease compared with other methods, further demonstrating the noise immunity of the proposed method. The accuracy, precision and recall of the presented method are better that $99 \%$ at $6 \mathrm{~dB}$ signal-to-noise ratio. In addition, the average test time for the three scenarios is $0.081 \mathrm{~s}$, indicating the good real-time performance of the presented method.

\section{Conclusion}

In this paper, a convolutional attention autoencoder mining hoist bearing fault diagnosis method is proposed, and a detailed theoretical analysis and comprehensive experimental verification are carried out. The main work and conclusions are as follows.

(1) Designing the channel attention module to extract the fault signal features and adding the idea of residual learning to solve the network degradation problem caused by the model becoming deeper, so that the network can learn the key features of the data more comprehensively.

(2) Build a convolutional autoencoder and combine the channel attention module to build a convolutional attention autoencoder feature extraction module to extract the fault features of the original input data; and 
design a fault feature classifier to classify the extracted features for diagnosis.

(3) The convolutional attention autoencoder fault diagnosis method has higher accuracy and noise immunity compared with other networks and can diagnose fault types in real time.

In the future, the network structure optimization of the convolutional attention autoencoder will be carried out to further investigate the application to the fault diagnosis method.

\section{Conflict of interest}

The authors declare that they have no known competing financial interests or personal relationships that could have appeared to influence the work reported in this paper.

\section{Open Access}

This article is licensed under a Creative Commons Attribution 4.0 International License, which permits use, sharing, adaptation, distribution and reproduction in any medium or format, as long as you give appropriate credit to the original author(s) and the source, provide a link to the Creative Commons licence, and indicate if changes were made. The images or other third party material in this article are included in the article's Creative Commons licence, unless indicated otherwise in a credit line to the material. If material is not included in the article's Creative Commons licence and your intended use is not permitted by statutory regulation or exceeds the permitted use, you will need to obtain permission directly from the copyright holder. To view a copy of this licence, visit http://creativecommons. org/licenses/by/4.0/.

\section{References}

He K, Wang Y, Xiao D (2016). Inhibition characteristics of circulating current in parallel inverter driving system of mine hoist. Int $\mathrm{J}$ Coal Sci Technol 3(1):68-76. https://doi.org/10.1007/ s40789-016-0104-7

Kou Z, Yang F, Wu J, Li T (2020). Application of ICEEMDAN Energy Entropy and AFSASVM for Fault Diagnosis of Hoist Sheave Bearing. Entropy 22(12): 1347. https://doi.org/10.3390/ e22121347

Jiang F, Zhu Z, Li W, Chen G, Zhou G (2013). Robust condition monitoring and fault diagnosis of rolling element bearings using improved EEMD and statistical features. Meas Sci Technol 25(2):025003. https : //doi.org/10.1088/0957-0233/25/2/025003

Gu J, Peng Y, Lu H, Cao S, Cao B (2020). Fault diagnosis of spindle device in hoist using variational mode decomposition and statistical features. Shock Vib. https://doi.org/10.1155/2020/8849513

Qi Y, Shen C, Wang D, Shi J, Jiang X, Zhu Z (2017). Stacked sparse autoencoder-based deep network for fault diagnosis of rotating machinery. IEEE Access 5:15066-15079. https://doi.org/10.1109/ACCESS . 2017.2728010

Hamadache M, Lee D (2017). Principal component analysis based signal-to-noise ratio improvement for inchoate faulty signals: Application to ball bearing fault detection. International Journal of Control. Int J Control Autom 15(2):506-517. https://doi.org/ 10.1007/s12555-015-0196-7

Eren L, Ince T, Kiranyaz S (2019). A generic intelligent bearing fault diagnosis system using compact adaptive 1D CNN classifier. J Signal Process Syst 91(2):179-189. https://doi.org/10. 1007/s11265-018-1378-3

Wang D, Guo Q, Song Y, Gao S, Li Y (2019). Application of multiscale learning neural network based on CNN in bearing fault diagnosis. J Signal Process Syst 91(10):1205-1217. https://doi .org/10.1007/ s11265-019-01461-w

Chen Z, Li W (2017). Multisensor feature fusion for bearing fault diagnosis using sparse autoencoder and deep belief network. IEEE Trans Instrum Meas 66(7):1693-1702. https://doi.org/10.1109/ TIM. 2017.2669947

Ma M, Sun C, Chen X (2018). Deep coupling autoencoder for fault diagnosis with multimodal sensory data. IEEE Trans Industr Inform 14(3):1137-1145. https://doi.org/10.1109/TII . 2018.2793246

Liu Z, Wang H, Liu J, Qin Y, Peng D (2020). Multitask learning based on lightweight 1DCNN for fault diagnosis of wheelset bearings. IEEE Trans Instrum Meas 70:1-11. https://doi.org/10.1109/ TIM. 2020.3017900

Wen L, Li X, Gao L, Zhang Y (2017). A new convolutional neural network-based data-driven fault diagnosis method. IEEE Trans Ind Electron 65(7):5990-5998. https://doi.org/10.1109/ TIE. 2017.2774777

Liu X, Zhou Q, Zhao J, Shen H, Xiong X (2019). Fault diagnosis of rotating machinery under noisy environment conditions based on a 1-D convolutional autoencoder and 1-D convolutional neural network. Sensors 19(4):972. https://doi.org/10. $3390 /$ s19040972 
Che C, Wang H, Ni X, Fu Q (2020). Intelligent fault diagnosis method of rolling bearing based on stacked denoising autoencoder and convolutional neural network. Ind Lubr Tribol. https://doi.org/10.1108/ ILT-11-2019-0496

Shao H, Jiang H, Zhao H, Wang F (2017). A novel deep autoencoder feature learning method for rotating machinery fault diagnosis. Mech Syst Signal Process 95:187-204. https://doi.org/10.1016/j. ymssp. 2017.03.034

Fu J, Liu J, Tian H, Li Y, Bao Y, Fang Z, Lu H (2019). Dual attention network for scene segmentation. Proc IEEE Comput Soc Conf Comput Vision Pattern Recognit 3146-3154. https://doi.org/10. 1109/CVPR.2019.00326

Xu K, Li S, Jiang X, Lu J, Yu T, Li R (2021). A novel transfer diagnosis method under unbalanced sample based on discrete-peak joint attention enhancement mechanism. Knowl-Based Syst 212:106645. https: //doi.org/10.1016/j.knosys.2020.106645

Wang H, Liu Z, Peng D, Qin Y (2019). Understanding and learning discriminant features based on multiattention 1DCNN for wheelset bearing fault diagnosis. IEEE Trans Industr Inform 16(9):5735-5745. https://doi.org/10.1109/TII. 2019.2955540

LOPARO K A. Case Western Reserve University Bearing Data Center[DB/OL]. http: //csegroups.case.edu/bearingdatacenter/ pages/download-data-file

Zhang $\mathrm{X}$, Han $\mathrm{P}, \mathrm{Xu}$ L, Zhang $\mathrm{F}$, Wang $\mathrm{Y}$, Gao L (2020). Research on Bearing Fault Diagnosis of Wind Turbine Gearbox Based on 1DCNN-PSOSVM. IEEE Access, 8:192248-192258. https://doi. org/10.1109/ACCESS . 2020.3032719

Zheng J, Jiang Z, Pan H (2018). Sigmoid-based refined composite multiscale fuzzy entropy and t-SNE based fault diagnosis approach for rolling bearing. Measurement 129:332-342. https://doi.org/10.1016/ j.measurement.2018.07.045 